\title{
Numerical growth of emittance in simulations of laser-wakefield acceleration
}

\author{
R. Lehe, ${ }^{*}$ A. Lifschitz, C. Thaury, and V. Malka \\ Laboratoire d'Optique Appliquée, ENSTA-CNRS-Ecole Polytechnique, UMR 7639, 91761 Palaiseau, France
}

X. Davoine

Commissariat a l'Energie Atomique, DAM, DIF, 91297 Arpajon, France

(Received 20 December 2012; published 28 February 2013)

\begin{abstract}
Transverse emittance is a crucial feature of laser-wakefield accelerators, yet accurately reproducing its value in numerical simulations remains challenging. It is shown here that, when the charge of the bunch exceeds a few tens of picocoulombs, particle-in-cell (PIC) simulations erroneously overestimate the emittance. This is mostly due the interaction of spurious Cherenkov radiation with the bunch, which leads to a steady growth of emittance during the simulation. A new computational scheme is proposed, which is free of spurious Cherenkov radiation. It can be easily implemented in existing PIC codes and leads to a substantial reduction of the emittance growth.
\end{abstract}

DOI: 10.1103/PhysRevSTAB.16.021301

PACS numbers: 52.38.Kd, 41.60.Bq, 52.65.Rr, 41.75.- $\mathrm{i}$

\section{INTRODUCTION}

Over the past ten years, laser-wakefield accelerators have considerably evolved and are now able to produce quasimonoenergetic electron beams [1-3] with energy up to $1 \mathrm{GeV}$ [4]. This fast progress was made possible partly thanks to particle-in-cell (PIC) codes [5] and to their ability to reproduce or predict the properties of the experimental beams (e.g. total charge, mean energy, and energy dispersion).

Among the key properties of the beam, transverse emittance has attracted a growing interest lately, as several applications-including a prospective compact free electron laser [6]—require a very low emittance. Accordingly, considerable research effort is currently invested in decreasing the emittance of the beam, and unprecedently low values have indeed been measured in recent experiments [7-10]. In this context, an accurate calculation of emittance in PIC codes is essential in order to accompany and guide further research.

PIC simulations have certainly been able to predict very low emittances (of the order of $0.1 \mathrm{~mm} \mathrm{mrad}$ ) [9,11], yet these occurrences are restricted to cases where the charge of the bunch is very low $(\leq 1 \mathrm{pC})$. Here we demonstrate that, in the case of a bunch having a more typical charge [tens of picocoulombs (pC) or more], standard PIC codes tend to largely overestimate transverse emittance. This is due to the emittance spuriously growing from the beginning to the end of the acceleration. While numerical heating [12] is known to produce a similar growth, we

\footnotetext{
*remi.lehe@ensta.fr
}

Published by the American Physical Society under the terms of the Creative Commons Attribution 3.0 License. Further distribution of this work must maintain attribution to the author(s) and the published article's title, journal citation, and DOI. find that, in our case, the dominating effect is the interaction of the bunch with numerical Cherenkov radiation.

Numerical Cherenkov radiation is a known effect in the PIC community [13]. It is associated with the finite difference time domain Yee scheme [14] and with the fact that, in this scheme, the numerical velocity of electromagnetic waves in vacuum is lower than the speed of light. As a result, macroparticles may travel faster than these waves and emit unphysical high-frequency Cherenkov radiation. Several methods have been proposed in other contexts, in order to mitigate this effect. These methods include lowpass filters [15-17] and modified computational scheme [16-19], for instance. However, none of these methods is ideally suited to the specificities of laser-wakefield simulations. We therefore propose a new computational scheme that is easily implemented in existing PIC codes, efficiently suppresses Cherenkov radiation, and considerably reduces the spurious growth of emittance.

The paper is organized as follows. In Sec. II, we consider a typical simulation of self-injection and report on the strong unphysical radiation that surrounds the electron bunch and the associated growth of emittance. Section III summarizes the main characteristics of Cherenkov radiation and shows that the radiation observed in the simulations is indeed of this type. Section IV proposes a computational scheme that prevents Cherenkov radiation. Finally, in Sec. V we discuss this new scheme and show that it leads to a reduced growth of emittance.

\section{GROWING EMITTANCE IN SIMULATIONS}

We begin by considering a typical simulation of laserwakefield acceleration. Parameters are chosen so as to lead to the self-injection of a few hundreds of $\mathrm{pC}$ of charge. More specifically, we consider a $1.5 \mathrm{~J}$ laser pulse $(\lambda=0.8 \mu \mathrm{m})$ with $35 \mathrm{fs}$ FWHM duration focused into a plasma with density $n=6 \times 10^{-3} n_{c}=1.0 \times 10^{19} \mathrm{~cm}^{-3}$ 
(where $n_{c}=1.75 \times 10^{21} \mathrm{~cm}^{-3}$ is the critical density at this wavelength). The laser is focused so that its waist in the focal plane would have been $17 \mu \mathrm{m}$ in vacuum, corresponding to $a_{0}=2$ (where $a_{0}$ is the peak value of the normalized vector potential). Notice that, due to selffocusing, the actual waist is slightly lower and $a_{0}$ is somewhat higher. The simulation was run using the fully 3D PIC code CALDER [20], which uses the Yee scheme and an iterative Poisson solver when solving the Maxwell equations. Calculations are performed in a moving window using $1600 \times 320 \times 320$ grid points, with grid spacing $\Delta x=0.032 \mu \mathrm{m}$ longitudinally and $\Delta y=\Delta z=0.25 \mu \mathrm{m}$ transversely, and using a time step $c \Delta t=0.96 \Delta x$ that is below the Courant-Friedrichs-Lewy (CFL) limit.

As the laser pulse propagates through the plasma, selfinjection occurs, leading to continuous trapping of background electrons. At a distance of $300 \mu \mathrm{m}$ from the point where injection started, the electron bunch has a total charge of about $250 \mathrm{pC}$, with a peak in the energy spectrum around $120 \mathrm{MeV}$ containing about $150 \mathrm{pC}$ (see the top panel of Fig. 1).

We observed that the emittance of the bunch steadily increases during the acceleration (see the lower panel of Fig. 1). In Fig. 1 and in the rest of this paper, the emittance calculated is the normalized emittance, which is defined, in the
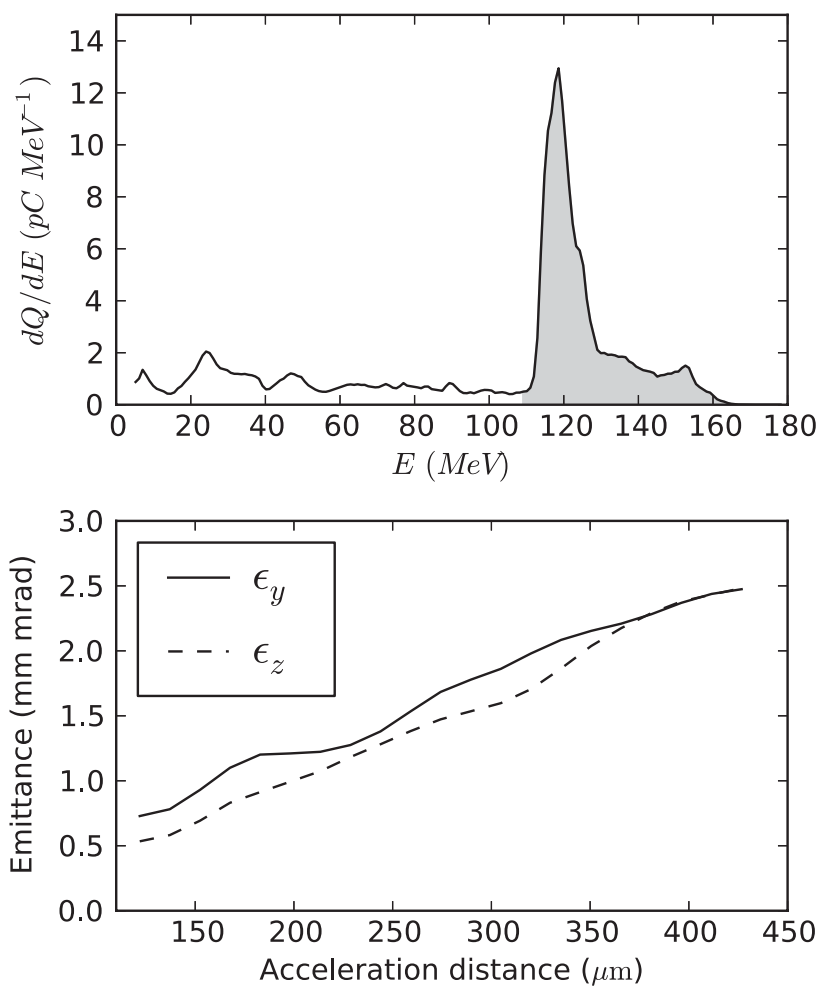

FIG. 1. Top: energy spectrum after $300 \mu \mathrm{m}$ of acceleration (from the position when injection started). The grayed area represents the sub-bunch for which the emittance was computed. Bottom: transverse emittance of this sub-bunch in the $y$ (parallel to the laser polarization) and $z$ (orthogonal to the laser polarization) direction, as a function of the acceleration distance. $y$ direction for instance, as $\epsilon_{y}=\sqrt{\left\langle y^{2}\right\rangle\left\langle p_{y}^{2}\right\rangle-\left\langle y p_{y}\right\rangle^{2}} /(m c)$, where brackets denote an average over the electron bunch and where $p_{y}=\gamma m \dot{y}$. It should be noted that in an fully evacuated plasma bubble [21,22], the transverse fields are linear functions of the radius, and as a result, normalized emittance should remain constant during the acceleration. On the contrary, the growth of emittance observed here is by no means negligible and reaches an average slope of about $6 \mathrm{~mm}$ mrad per $\mathrm{mm}$ of acceleration.

In the same simulation, we also observed that the bunch is surrounded by a strong high-frequency radiation. Figure 2 is a snapshot of the bubble after $300 \mu \mathrm{m}$ of acceleration and shows this radiation very clearly. Notice that this figure is a representation of $E_{y}-c B_{z}$ instead of $E_{y}$. The reason for this is that $E_{y}-c B_{z}$ is proportional to the Lorentz force felt by the electrons $F_{y}=$ $-e\left(E_{y}-v_{x} B_{z}+v_{z} B_{x}\right) \approx-e\left(E_{y}-c B_{z}\right)$. Moreover, a plot of $E_{y}$ would be dominated by the strong space-charge electric field of the bunch, whereas its effects are known to be almost canceled by the corresponding $\vec{v} \times \vec{B}$ term.

Importantly, the same effects (growth of emittance and simultaneous radiation around the bunch) also appeared in simulations of colliding-pulse injection-although not shown here-in which the charge of the bunch was $50 \mathrm{pC}$ and the emittance increased by $2 \mathrm{~mm}$ mrad per $\mathrm{mm}$ of acceleration. In both cases, the observed growth of emittance has serious consequences for the interpretation of the simulation, and it is of paramount importance to determine whether its origin is numerical or physical. Here we show that the growth of emittance is largely due to the observed radiation, which scatters electrons in the transverse direction by the means of the Lorentz force. This

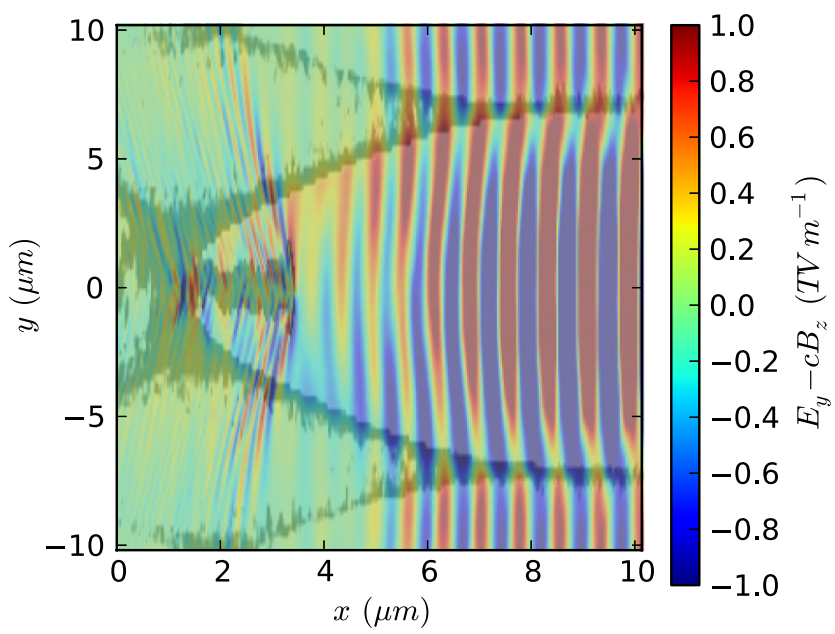

FIG. 2. Snapshot of the transverse field $E_{y}-c B_{z}$ in the $x-y$ plane, at the back of the bubble and after $300 \mu \mathrm{m}$ of acceleration. Shaded regions correspond to zones of high electron density (in this case the sheath of the bubble and the accelerated bunch). While the laser pulse is clearly apparent on the right-hand side of the figure, one also notices higher-frequency radiation around the bunch. 
effect is indeed suggested by a close examination of the bunch in Fig. 2, which reveals transverse oscillations of its structure having the same spatial frequency as that of the radiation. The same effect will be confirmed in Sec. V, where the radiation is intentionally suppressed and the growth of emittance is observed to be slower.

\section{NUMERICAL CHERENKOV RADIATION}

It seems very likely that the radiation seen around the bunch is produced by the bunch itself, and a reasonable mechanism for this is the numerical Cherenkov effect. In this section, we summarize the theoretical properties of numerical Cherenkov radiation and we show that the radiation seen here is indeed of this type.

Cherenkov radiation [23] - whether physical or numerical-may appear when the dispersion relation of electromagnetic waves allows for some modes to travel with a phase velocity lower than the speed of light $\left(v_{\phi}<c\right)$. In this case, energetic charged particles traveling close to the speed of light can excite some of those modes, thus producing a characteristic radiation. In the case of a charged particle traveling, e.g., along the $x$ axis with speed $\beta c(\beta<1)$, the modes that are excited are those that satisfy the relation

$$
\beta c=v_{\phi, x},
$$

where $v_{\phi, x}$ is the phase velocity of the mode along the $x$ axis. In particular, $v_{\phi, x}=\omega / k_{x}$ for harmonic waves of the form $\exp (i \vec{k} \cdot \vec{r}-i \omega t)$.

For electromagnetic waves traveling in vacuum the dispersion relation is $\omega^{2}=c^{2} \vec{k}^{2}$, and therefore $v_{\phi, x}>\beta c$. As a result, Eq. (1) cannot be satisfied and no Cherenkov emission occurs. This is shown in Fig. 3 where the plot of $v_{\phi, x}$ and the plane corresponding to $\beta c$ do not intersect. However, due to

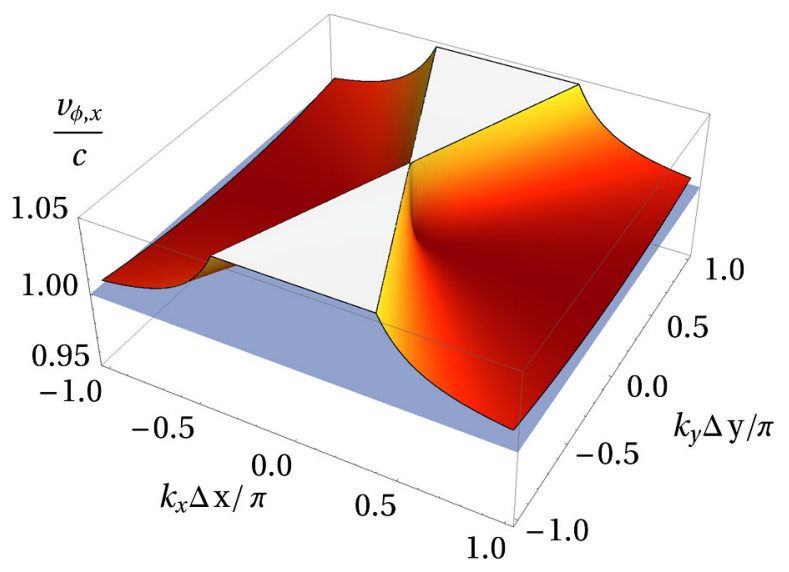

FIG. 3. Physical phase velocity along $x\left(v_{\phi, x}\right.$, as deduced from $\left.\omega^{2}=c^{2} \vec{k}^{2}\right)$ as a function of $k_{x}$ and $k_{y}$ for electromagnetic modes propagating in the $x-y$ plane $\left(k_{z}=0\right)$. The blue horizontal plane corresponds to $v_{\phi, x}=\beta c$ with $\beta=1-9 \times 10^{-6}$ (which is representative of particles having an energy of $120 \mathrm{MeV}$ ).

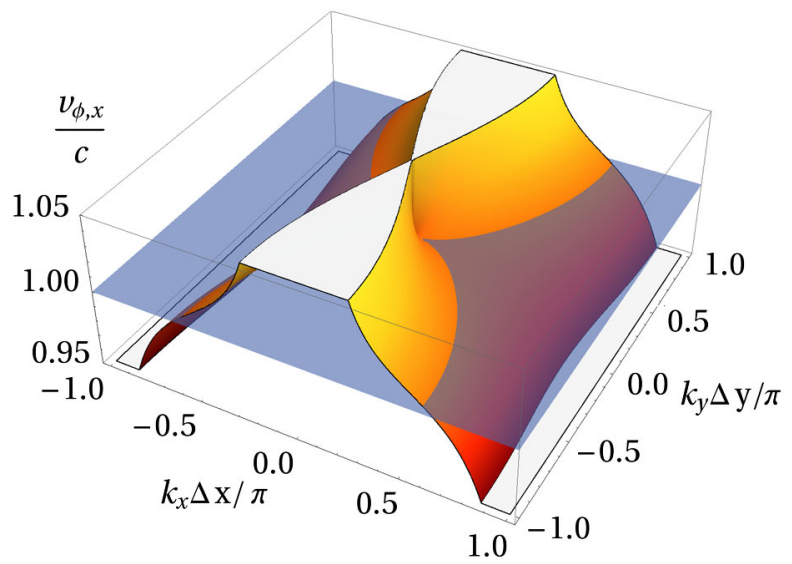

FIG. 4. Numerical phase velocity along $x\left[v_{\phi, x}\right.$, as deduced from Eq. (2)] as a function of $k_{x}$ and $k_{y}$ for electromagnetic modes propagating in the $x-y$ plane $\left(k_{z}=0\right)$. The blue horizontal plane corresponds to $v_{\phi, x}=\beta c$ with $\beta=1-9 \times 10^{-6}$ (which is representative of particles having an energy of $120 \mathrm{MeV}$ ).

spatial and temporal discretization in the Yee scheme, the actual numerical dispersion relation of these waves is

$$
\begin{aligned}
\frac{1}{c^{2} \Delta t^{2}} \sin ^{2}\left(\frac{\omega \Delta t}{2}\right)= & \frac{1}{\Delta x^{2}} \sin ^{2}\left(\frac{k_{x} \Delta x}{2}\right)+\frac{1}{\Delta y^{2}} \sin ^{2}\left(\frac{k_{y} \Delta y}{2}\right) \\
& +\frac{1}{\Delta z^{2}} \sin ^{2}\left(\frac{k_{z} \Delta z}{2}\right),
\end{aligned}
$$

and thus some values of $\omega$ and $\vec{k}$ may satisfy Eq. (1) in this case. This is shown in Fig. 4 for modes propagating in the $x-y$ plane. The modes that are predicted to be excited by numerical Cherenkov effect are those for which the two

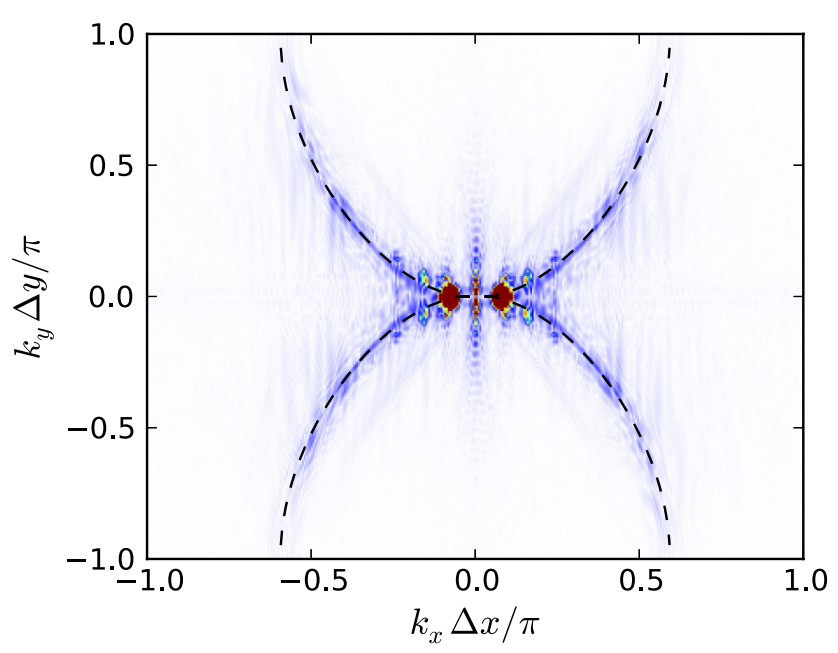

FIG. 5. Spatial Fourier transform of the field $E_{y}-c B_{z}$ from Fig. 2. The two red spots correspond to the laser field $\left(k_{x} \Delta x / \pi=\right.$ $\left.\pm k_{\text {laser }} \Delta x / \pi= \pm 0.08\right)$. The structures observed near $k_{x}=0$ correspond to the fields of the bubble (their broad extent along $k_{y}$ in Fourier space corresponds to the sharp edges of the bubble in real space). The dashed line materializes the position of the modes satisfying Eq. (3) (with $\beta=1-9 \times 10^{-6}$ ) and thus corresponds to the intersection of the two theoretical surfaces of Fig. 4. 
surfaces of Fig. 4 intersect. Their equation in the $k_{x}-k_{y}$ plane is thus

$$
\begin{aligned}
& \frac{1}{c^{2} \Delta t^{2}} \sin ^{2}\left(\frac{\beta c k_{x} \Delta t}{2}\right) \\
& =\frac{1}{\Delta x^{2}} \sin ^{2}\left(\frac{k_{x} \Delta x}{2}\right)+\frac{1}{\Delta y^{2}} \sin ^{2}\left(\frac{k_{y} \Delta y}{2}\right)+\frac{1}{\Delta z^{2}} \sin ^{2}\left(\frac{k_{z} \Delta z}{2}\right) .
\end{aligned}
$$

In order to prove that the radiation observed in Sec. II is due to numerical Cherenkov effect, we verify that it corresponds to modes satisfying Eq. (3). To this end, we perform the spatial Fourier transform of the field $E_{y}-c B_{z}$ in the $x-y$ plane of our simulation and show the result in Fig. 5. As can be seen, the only modes with sizeable amplitude are exactly those that are predicted by Eq. (3) to be excited through numerical Cherenkov effect. We conclude that this radiation is indeed of numerical origin. We will now show that it can be removed by using a modified numerical scheme.

\section{A PROPOSED SCHEME TO AVOID NUMERICAL CHERENKOV RADIATION}

A number of solutions have already been proposed in order to avoid numerical Cherenkov effect, albeit in different contexts (including for instance the case of boosted-frame simulations of laser-wakefield acceleration [17]). Yet, none of them is well suited to model laserwakefield acceleration in the laboratory frame.

For instance, some of these solutions [15-17] consist of using digital filtering, either in space or time, in order to damp high-frequency radiation. However, any filtering operation in real space (as opposed to Fourier space) that strongly damps high-frequency radiation inevitably also affects the physics at lower frequency. The effect of such a filter is particularly deleterious for physical phenomena having frequencies close to those that are to be filtered. In the case of laser-wakefield acceleration, the laser has a relatively high frequency, which makes it impossible to filter Cherenkov radiation without damping the laser itself-especially if the filter has to be applied at each time step and if the laser propagates over large distances. A notable exception to this rule is the case of boostedframe simulations [24], in which the laser-as seen in a proper relativistic frame-has a low spatial frequency [25], which then allows aggressive filtering.

Another set of solutions consist of modifying the discretization of the Maxwell equations, which then modifies the dispersion relation of electromagnetic modes. In $[18,19]$, the numerical scheme is modified so that the CFL condition allows one to choose $c \Delta t=\Delta x$. In this case, the dispersion relation is exact for modes propagating along the $x$ axis, and Cherenkov radiation can potentially be suppressed. However, it was shown in [17] that using $c \Delta t=\Delta x$ triggers the onset of spurious numerical oscillations at the Nyquist frequency $(k=\pi / \Delta x)$. Again, strong filtering is needed in this case, in order to remove these oscillations. Along the same line of thought, another set of numerical schemes was proposed in [16], and some of them can prevent numerical Cherenkov radiation without requiring $c \Delta t=\Delta x$. These methods could in principle be successfully used in laser-wakefield simulation, yet they were only developed for isotropic grids $(\Delta x=\Delta y=\Delta z$ ), which limits their range of application. In this section, we propose a variation of the schemes proposed in [16], which is optimally adapted to simulations of laser-wakefield acceleration. Importantly, the proposed scheme is applicable to anisotropic grids and minimizes the impact of the modified dispersion relation on the propagation of the laser.

\section{A. Description of the proposed scheme}

In the proposed scheme, the fields $\vec{E}$ and $\vec{B}$ are defined on the same lattices as in the Yee scheme. The Maxwell equations take the following form:

$$
\begin{gathered}
D_{t} \vec{B}=-\vec{\nabla}^{*} \times \vec{E}, \\
D_{t} \vec{E}=c^{2} \vec{\nabla} \times \vec{B}-\mu_{0} c^{2} \vec{J},
\end{gathered}
$$

where $D_{t}$ and $\vec{\nabla}$ symbolize the same discretized leap -frog operators as in the Yee scheme, i.e., for a field $F_{i, j, k}^{n}$ defined on the nodes of the computational lattice, $\left.D_{t} F\right|_{i, j, k} ^{n+1 / 2}=\left(F_{i, j, k}^{n+1}-F_{i, j, k}^{n}\right) / \Delta t$ and $\left.\nabla_{x} F\right|_{i+1 / 2, j, k} ^{n}=$ $\left(F_{i+1, j, k}^{n}-F_{i, j, k}^{n}\right) / \Delta x$. However, $\vec{\nabla}^{*}$ is a modified operator that depends on a set of coefficients, and which is defined by

$$
\begin{aligned}
\left.\nabla_{x}^{*} F\right|_{i+1 / 2, j, k} ^{n}= & \alpha_{x}\left(F_{i+1, j, k}^{n}-F_{i, j, k}^{n}\right) / \Delta x+\beta_{x, y}\left(F_{i+1, j+1, k}^{n}-F_{i, j+1, k}^{n}\right) / \Delta x+\beta_{x, y}\left(F_{i+1, j-1, k}^{n}-F_{i, j-1, k}^{n}\right) / \Delta x \\
& +\beta_{x, z}\left(F_{i+1, j, k+1}^{n}-F_{i, j, k+1}^{n}\right) / \Delta x+\beta_{x, z}\left(F_{i+1, j, k-1}^{n}-F_{i, j, k-1}^{n}\right) / \Delta x+\delta_{x}\left(F_{i+2, j, k}^{n}-F_{i-1, j, k}^{n}\right) / \Delta x
\end{aligned}
$$

and by similar relations along the directions $y$ and $z$. The position of the nodes that are used to calculate $\left.\nabla_{x}^{*} F\right|_{i+1 / 2, j, k} ^{n}$ are represented in Fig. 6. In order for the operator $\nabla_{x}^{* ;, t o}$ reduce to $\partial_{x}$ in the limit $\Delta x \rightarrow 0$, the following relation has to be imposed:

$$
\alpha_{x}=1-2 \beta_{x, y}-2 \beta_{x, z}-3 \delta_{x} .
$$

Similar relations also have to be imposed in the directions $y$ and $z$.

By injecting functions of the form $\vec{E}, \vec{B} \propto \exp (i \vec{k} \cdot \vec{x}-\omega t)$ into the discretized Maxwell equations, it is possible to show that the numerical dispersion relation in this scheme is 


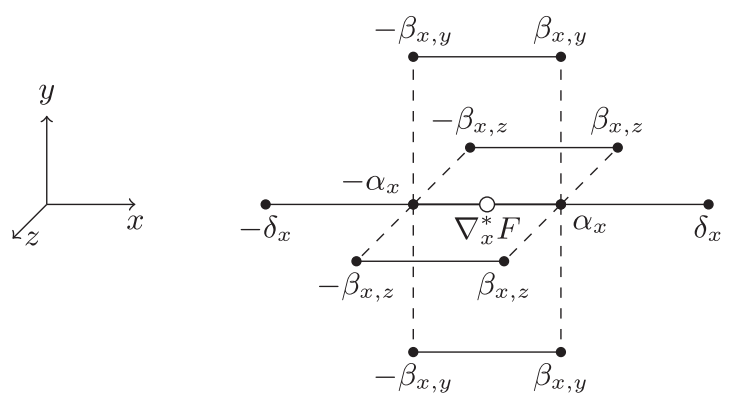

FIG. 6. Position of the nodes used to compute $\left.\nabla_{x}^{*} F\right|_{i+1 / 2, j, k} ^{n}$ in our modified scheme.

$$
\begin{aligned}
\frac{s_{t}^{2}}{c^{2} \Delta t^{2}}= & \frac{s_{x}^{2}}{\Delta x^{2}}+\frac{s_{y}^{2}}{\Delta y^{2}}+\frac{s_{z}^{2}}{\Delta z^{2}} \\
& -4\left(\delta_{x} \frac{s_{x}^{4}}{\Delta x^{2}}+\delta_{y} \frac{s_{y}^{4}}{\Delta y^{2}}+\delta_{z} \frac{s_{z}^{4}}{\Delta z^{2}}\right) \\
& -4\left(\frac{1}{\Delta x^{2}} \beta_{x, y}+\frac{1}{\Delta y^{2}} \beta_{y, x}\right) s_{x}^{2} s_{y}^{2} \\
& -4\left(\frac{1}{\Delta y^{2}} \beta_{y, z}+\frac{1}{\Delta z^{2}} \beta_{z, y}\right) s_{y}^{2} s_{z}^{2} \\
& -4\left(\frac{1}{\Delta z^{2}} \beta_{z, x}+\frac{1}{\Delta x^{2}} \beta_{x, z}\right) s_{z}^{2} s_{x}^{2}
\end{aligned}
$$

with $s_{t}=\sin (\omega \Delta t / 2)$ and $s_{u}=\sin \left(k_{u} \Delta u / 2\right)$ for $u=x, y$, $z$. Selecting an appropriate scheme now reduces to the choice of the $\beta$ and $\delta$ coefficients.

In order to determine the coefficients that efficiently suppress Cherenkov radiation, we first impose that the phase velocity of modes propagating along the $x$ axis is larger than $c\left[v_{\phi, x}\left(\vec{k}=k_{x} \vec{u}_{x}\right) \geq c\right.$, for any $k_{x}$, where $\vec{u}_{x}$ is the unit vector along $x$ ]. From Eq. (8), the phase velocity of such modes is

$v_{\phi, x}\left(\vec{k}=k_{x} \vec{u}_{x}\right)=\frac{2}{k_{x} \Delta t} \arcsin \left(\frac{c \Delta t}{\Delta x} s_{x} \sqrt{1-4 \delta_{x} s_{x}^{2}}\right)$.

Thus, for a given $\Delta t$ and $\Delta x, v_{\phi, x}$ increases when $\delta_{x}$ decreases (even for negative values of $\delta_{x}$ ). In fact, $v_{\phi, x}$ is larger than $c$ for any $k_{x}$, when $\delta_{x} \leq \delta_{x, 0}$, where

$$
\delta_{x, 0}=\frac{1}{4}\left[1-\frac{\Delta x^{2}}{c^{2} \Delta t^{2}} \sin ^{2}\left(\frac{\pi c \Delta t}{2 \Delta x}\right)\right] .
$$

Notice, incidentally, that $\delta_{x}$ should not be chosen much below $\delta_{x, 0}$, since CFL-type instabilities start appearing for $\delta_{x}<1 / 4\left[1-\Delta x^{2} /(c \Delta t)^{2}\right]$. In the proposed algorithm, we opt for $\delta_{x}=\delta_{x, 0}$.

However, implementing $\delta_{x}=\delta_{x, 0}$ also affects modes having an oblique $\vec{k}$, and makes them more prone to CFL instabilities. Adopting proper nonzero $\beta$ coefficients stabilizes these modes and efficiently prevents CFL instabilities. In the end, the proposed scheme corresponds to the following coefficients:

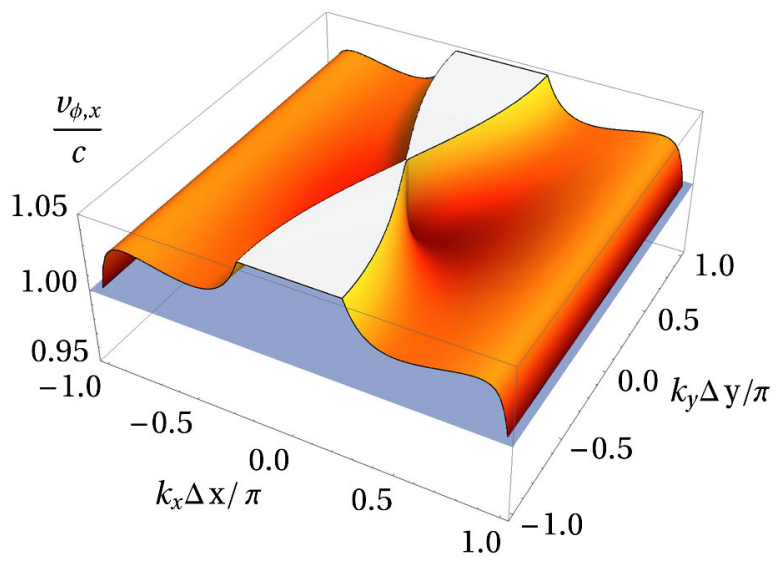

FIG. 7. Numerical phase velocity along $x\left[v_{\phi, x}\right.$, as deduced from Eq. (8)] as a function of $k_{x}$ and $k_{y}$ for electromagnetic modes propagating in the $x-y$ plane $\left(k_{z}=0\right)$. The blue horizontal plane corresponds to $v_{\phi, x}=\beta c$, and there is no intersection between these two surfaces. Although not shown on this figure, there is no intersection either for the other modes $\left(k_{z} \neq 0\right)$.

$$
\begin{aligned}
\beta_{y, x} & =\beta_{z, x}=1 / 8, \quad \beta_{z, y}=\beta_{y, z}=0, \\
\beta_{x, y} & =\Delta x^{2} /\left(8 \Delta y^{2}\right), \quad \beta_{x, z}=\Delta x^{2} /\left(8 \Delta z^{2}\right), \\
\delta_{x} & =\delta_{x, 0}, \quad \delta_{y}=\delta_{z}=0 .
\end{aligned}
$$

As long as $c \Delta t \leq \Delta x$, this scheme is CFL stable. Moreover, it satisfies $v_{\phi, x} \geq c$ for all modes (see Fig. 7), thus preventing Cherenkov radiation for particles traveling along the $x$ direction. We emphasize that this scheme is only efficient in the cases where relativistic particles all travel along the same axis. This is, however, clearly the case in simulations of laser-wakefield acceleration.

\section{B. Spurious oscillations at the Nyquist frequency and velocity of the laser}

We point out that, in the proposed scheme, the ratio $c \Delta t / \Delta x$ is a free parameter (on which the coefficient $\delta_{x}$ depends). Choosing $c \Delta t / \Delta x=1$ leads to $\delta_{x}=0$, and the scheme reduces to that of [19]. However, as observed in [17], this scheme develops spurious oscillations at the Nyquist frequency, which-unless filtered-rapidly grow to unacceptable levels. We observed that reducing $c \Delta t / \Delta x$ in our scheme readily leads to a reduced level of oscillations. In practice, even for $c \Delta t / \Delta x=0.96$, these oscillations remain at a limited level instead of growing unbound, so that no filtering is required in the simulation.

Another important point to bear in mind is that the proposed scheme artificially increases the velocity of the laser-in the same way as the Yee scheme artificially reduces it. In the limit $k_{\text {laser }} \Delta x \ll 1$ and $1-c \Delta t / \Delta x \ll$ 1 , Eq. (9) with $\delta_{x}=\delta_{x, 0}$ reduces to

$$
v_{\phi, x}\left(\vec{k}_{\text {laser }}\right) / c=1+\frac{2}{3}\left(1-\frac{c \Delta t}{\Delta x}\right)\left(\frac{k_{\text {laser }} \Delta x}{2}\right)^{2},
$$


which also implies

$$
v_{g, x}\left(\vec{k}_{\text {laser }}\right) / c=1+2\left(1-\frac{c \Delta t}{\Delta x}\right)\left(\frac{k_{\text {laser }} \Delta x}{2}\right)^{2},
$$

where $v_{g, x} \equiv \frac{\partial \omega}{\partial k_{x}}=\frac{\partial}{\partial k_{x}}\left(k_{x} v_{\phi, x}\right)$ is the group velocity of the laser along $x$.

The laser speedup here is of the same order of magnitude as the laser slowdown in the Yee scheme, since $v_{\phi, x}^{\mathrm{Yee}}\left(\vec{k}_{\text {laser }}\right) / c=1-1 / 3 \times(1-c \Delta t / \Delta x)\left(k_{\text {laser }} \Delta x / 2\right)^{2}$ and

$v_{g, x}^{\text {Yee }}\left(\vec{k}_{\text {laser }}\right) / c=1-(1-c \Delta t / \Delta x)\left(k_{\text {laser }} \Delta x / 2\right)^{2}$. When simulating laser-wakefield acceleration, it is important to ensure that the numerical alteration of the laser group velocity is negligible compared to the physical alteration caused by the plasma. In other terms, the criterion $(1-c \Delta t / \Delta x)\left(k_{\text {laser }} \Delta x / 2\right)^{2} \ll\left(n / n_{c}\right)$ has to be satisfied. Notice that this holds for both schemes. Not satisfying the above criteria would lead, in the Yee scheme, to the injection of a spuriously high level of charge, while it would lead, in the proposed scheme, to an artificially low charge or no injection at all.

Satisfying the above criterion implies that the laser wavelength has to be sufficiently resolved (i.e. $k_{\text {laser }} \Delta x$ sufficiently small) but also that $c \Delta t / \Delta x$ should not be too different from 1 . There is therefore a trade-off between $c \Delta t / \Delta x$ far from 1 , which keeps numerical oscillations low and $c \Delta t / \Delta x$ close to 1 , which reduces the alteration of the velocity of the laser.

\section{SUPPRESSION OF CHERENKOV RADIATION}

We implemented the scheme proposed in Sec. IV in CALDER and ran a simulation with the same parameters as in Sec. II. In particular, we used $c \Delta t=0.96 \Delta x$. Figure 8 compares the field $E_{y}-c B_{z}$ obtained in those two simulations, after $300 \mu \mathrm{m}$ of acceleration. Consistently with the analysis of Sec. IV, no Cherenkov radiation is emitted in the case of the modified scheme. We emphasize that this is indeed a consequence of the dispersion relation of the proposed scheme, since no filtering was used in the simulation. On the other hand, oscillations at the Nyquist frequency appear behind the accelerated bunch in the case of the modified scheme. Nonetheless, these oscillations seem to have much less impact on the bunch than the Cherenkov radiation does. This can be seen, in particular, in Fig. 9, where the evolution of the transverse emittance is plotted in both cases. Suppressing the Cherenkov radiation allowed us to reduce the growth rate of emittance by roughly a factor of 2 . In simulations of controlled injection where the charge of the bunch was lower $(\simeq 50 \mathrm{pC})$, we were able to observe a reduction by a factor of 7 . This strong reduction proves that Cherenkov radiation is indeed the main cause for the growth of emittance. It is unclear whether the remaining growth of emittance is the consequence of a physical effect (such as a partially evacuated bubble) or of another numerical artifact.
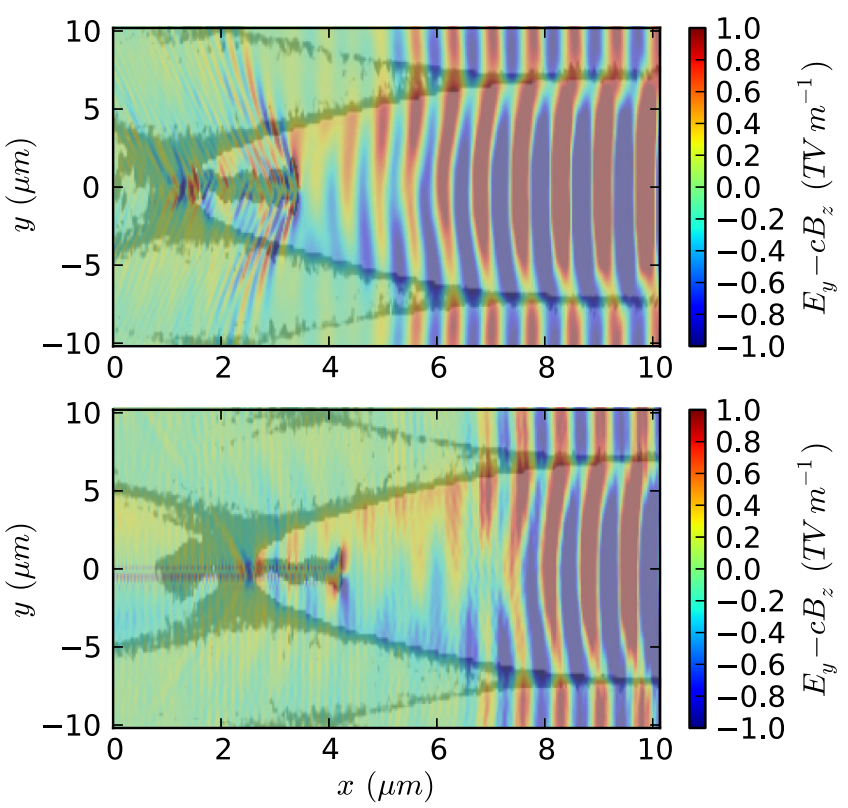

FIG. 8. Snapshot of the transverse field $E_{y}-c B_{z}$ in the $x-y$ plane, at the back of the bubble and after $300 \mu \mathrm{m}$ of acceleration. The top panel corresponds to the simulation ran with the Yee scheme, while the bottom panel corresponds to the one run with our modified scheme. Both snapshots correspond to the same iteration, so that the bubble has a slight advance in the bottom panel, due to the alteration of the velocity of the laser mentioned in Sec. IV B.
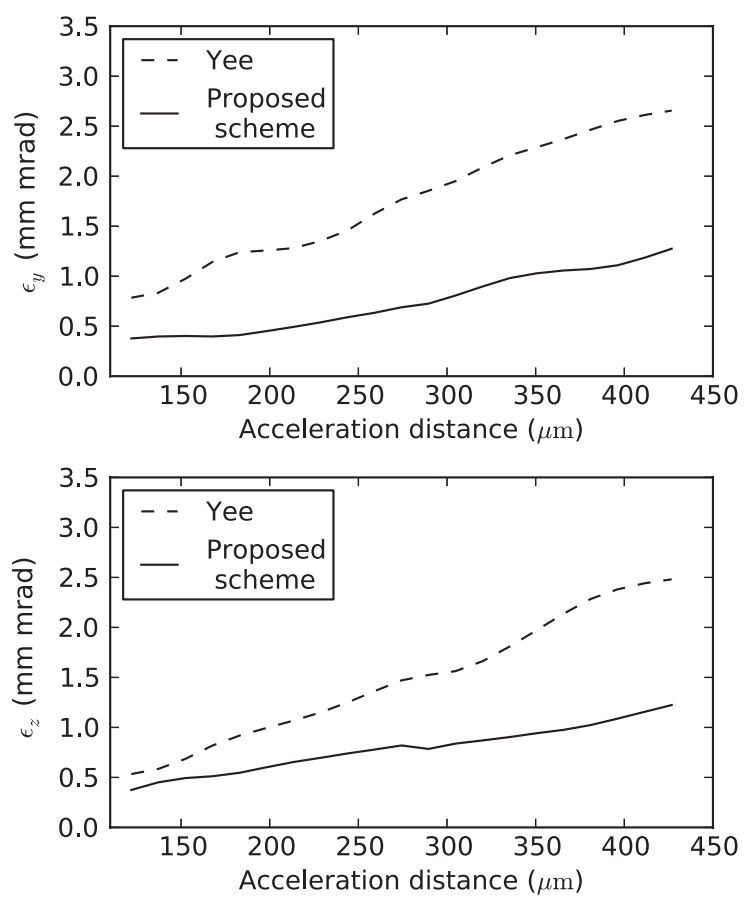

FIG. 9. Transverse emittance of the sub-bunch considered in Fig. 1, in the $y$ (parallel to the laser polarization) and $z$ (orthogonal to the laser polarization) direction, as a function of the acceleration distance. 


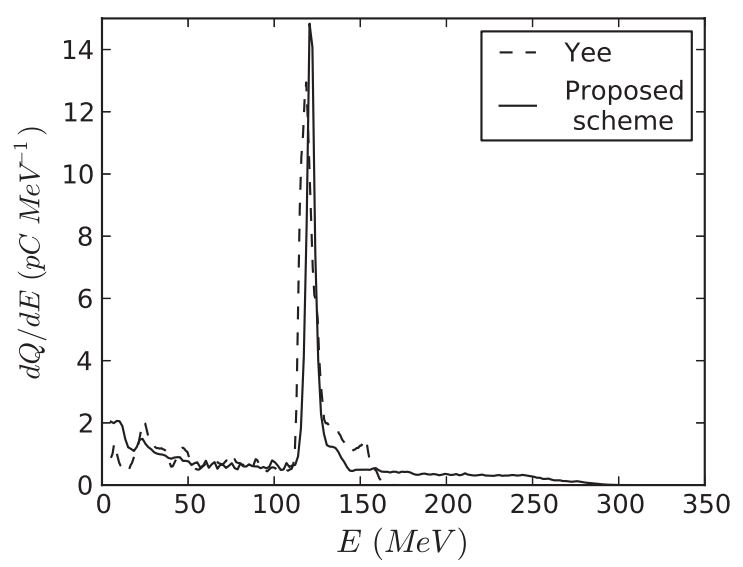

FIG. 10. Energy spectrum after $300 \mu \mathrm{m}$ of acceleration (from the position when injection started) for the two numerical schemes considered here.

The impact of the numerical scheme on the group velocity of the laser, which was mentioned in Sec. IV B, is also clearly visible in Fig. 8, where the laser pulse has a slight advance in the proposed scheme, as compared to the Yee scheme. In the two simulations, the laser pulse was initialized at the same position, but it propagated then over about $725 \mu \mathrm{m}$ before the snapshot from Fig. 8 was taken. From Eq. (13) and its counterpart for the Yee scheme, the expected difference in laser group velocity between the two schemes is $\Delta v_{g, x} / c=3(1-c \Delta t / \Delta x)\left(k_{\text {laser }} \Delta x / 2\right)^{2}=$ $1.9 \times 10^{-3}$. After $725 \mu \mathrm{m}$ of propagation, this leads to an expected advance of $1.4 \mu \mathrm{m}$ in the proposed scheme as compared to the Yee scheme, which is consistent with the observations in Fig. 8.

Apart from the differences mentioned above, our modified scheme did not affect significantly the physics in the simulation. In particular, the energy spectra of the electrons are very similar in the two simulations, as shown in Fig. 10, and the total charge of the bunch differs by less than $5 \%$ from one scheme to the other.

\section{CONCLUSION}

In this paper, we showed that simulations of laserwakefield acceleration using standard PIC codes tend to largely overestimate the emittance of the bunch. In addition, we proved that this overestimation is, to a large extent, due to numerical Cherenkov radiation interacting with the bunch. By using a modified numerical scheme, we were able to suppress Cherenkov radiation and substantially reduce the calculated emittance.

The main implication of this work is that calculations of emittance, with bunches of a few tens of $\mathrm{pC}$ and more, require much care. The low values of emittance, that are now reached in experiments, are indeed extremely sensitive to the numerical artifacts of PIC simulations. Although we removed an important fraction of these artifacts, more work is needed in order to determine wether the obtained emittance is free of numerical errors.

Another important contribution of this paper is the numerical scheme that we propose. This scheme efficiently suppresses Cherenkov radiation and yet develops only a limited level of high-frequency numerical oscillations, as compared to the scheme used in [17]. As a result, valid simulations can be run with our scheme, without the need of low-pass filters. This algorithm, or modified versions of it, could in principle be used in other contexts where the dispersion relation is crucial. This potentially includes simulations of relativistic shock formation [26,27], as well as simulations of laser-wakefield acceleration in the boosted frame [24,28], since Cherenkov-type instabilities can represent a serious issue in both cases.

\section{ACKNOWLEDGMENTS}

We are grateful to J. Faure for fruitful discussions. This work was partially supported by the European Research Council through the PARIS ERC project (Contract No. 226424).

[1] J. Faure, Y. Glinec, A. Pukhov, S. Kiselev, S. Gordienko, E. Lefebvre, J.-P. Rousseau, F. Burgy, and V. Malka, Nature (London) 431, 541 (2004).

[2] C. G. R. Geddes, C. Toth, J. van Tilborg, E. Esarey, C. B. Schroeder, D. Bruhwiler, C. Nieter, J. Cary, and W.P. Leemans, Nature (London) 431, 538 (2004).

[3] S.P.D. Mangles, C. D. Murphy, Z. Najmudin, A. G. R. Thomas, J. L. Collier, A.E. Dangor, E. J. Divall, P. S. Foster, J. G. Gallacher, C. J. Hooker, D. A. Jaroszynski, A. J. Langley, W. B. Mori, P. A. Norreys, F. S. Tsung, R. Viskup, B.R. Walton, and K. Krushelnick, Nature (London) 431, 535 (2004).

[4] W. P. Leemans, B. Nagler, A. J. Gonsalves, C. Toth, K. Nakamura, C. G. R. Geddes, E. Esarey, C. B. Schroeder, and S. M. Hooker, Nat. Phys. 2, 696 (2006).

[5] C. Birdsall and C. Langdon, Plasma Physics Via Computer Simulation, Series in Plasma Physics (Taylor \& Francis Group, New York, 2005).

[6] K. Nakajima, Nat. Phys. 4, 92 (2008).

[7] R. Weingartner, S. Raith, A. Popp, S. Chou, J. Wenz, K. Khrennikov, M. Heigoldt, A. R. Maier, N. Kajumba, M. Fuchs, B. Zeitler, F. Krausz, S. Karsch, and F. Grüner, Phys. Rev. ST Accel. Beams 15, 111302 (2012).

[8] S. Kneip, C. McGuffey, J.L. Martins, M.S. Bloom, V. Chvykov, F. Dollar, R. Fonseca, S. Jolly, G. Kalintchenko, K. Krushelnick, A. Maksimchuk, S.P.D. Mangles, Z. Najmudin, C. A. J. Palmer, K. T. Phuoc, W. Schumaker, L. O. Silva, J. Vieira, V. Yanovsky, and A. G. R. Thomas, Phys. Rev. ST Accel. Beams 15, 021302 (2012).

[9] G. R. Plateau, C. G. R. Geddes, D. B. Thorn, M. Chen, C. Benedetti, E. Esarey, A.J. Gonsalves, N.H. Matlis, K. Nakamura, C. B. Schroeder, S. Shiraishi, T. Sokollik, J. van Tilborg, C. Toth, S. Trotsenko, T.S. Kim, 
M. Battaglia, T. Stöhlker, and W. P. Leemans, Phys. Rev. Lett. 109, 064802 (2012).

[10] S. Corde, K. T. Phuoc, R. Fitour, J. Faure, A. Tafzi, J. P. Goddet, V. Malka, and A. Rousse, Phys. Rev. Lett. 107, 255003 (2011).

[11] T. Mehrling, J. Grebenyuk, F. S. Tsung, K. Floettmann, and J. Osterhoff, Phys. Rev. ST Accel. Beams 15, 111303 (2012).

[12] E. Cormier-Michel, B. A. Shadwick, C. G. R. Geddes, E. Esarey, C. B. Schroeder, and W. P. Leemans, Phys. Rev. E 78, 016404 (2008).

[13] B. B. Godfrey, J. Comput. Phys. 15, 504 (1974).

[14] K. Yee, IEEE Trans. Antennas Propag. 14, 302 (1966).

[15] A. D. Greenwood, K. Cartwright, E. Baca, and J. Luginsland, Antennas and Propagation Society International Symposium, 2002. IEEE, 3, 268 (2002).

[16] A. D. Greenwood, K. L. Cartwright, J. W. Luginsland, and E. A. Baca, J. Comput. Phys. 201, 665 (2004).

[17] J.-L. Vay, C. Geddes, E. Cormier-Michel, and D. Grote, J. Comput. Phys. 230, 5908 (2011).

[18] M. Karkkainen, E. Gjonaj, T. Lau, and T. Weiland, in Proceedings of ICAP 2006, Chamonix, France, Vol. 1
[Joint Accelerator Conferences Website (JACoW), Europe, 2006], p. 35.

[19] A. Pukhov, J. Plasma Phys. 61, 425 (1999).

[20] E. Lefebvre, N. Cochet, S. Fritzler, V. Malka, M.-M. Alonard, J.-F. Chemin, S. Darbon, L. Disdier, J. Faure, A. Fedotoff, O. Landoas, G. Malka, V. Mot, P. Morel, M.R.L. Gloahec, A. Rouyer, C. Rubbelynck, V. Tikhonchuk, R. Wrobel, P. Audebert, and C. Rousseaux, Nucl. Fusion 43, 629 (2003).

[21] I. Kostyukov, A. Pukhov, and S. Kiselev, Phys. Plasmas 11, 5256 (2004).

[22] W. Lu, C. Huang, M. Zhou, W. B. Mori, and T. Katsouleas, Phys. Rev. Lett. 96, 165002 (2006).

[23] J.D. Jackson, Classical Electrodynamics (Wiley, New York, 1998), 3rd ed.

[24] J.-L. Vay, Phys. Rev. Lett. 98, 130405 (2007).

[25] J.-L. Vay, C. G. R. Geddes, E. Cormier-Michel, and D.P. Grote, Phys. Plasmas 18, 030701 (2011).

[26] A. Spitkovsky, Astrophys. J. 682, L5 (2008).

[27] U. Keshet, B. Katz, A. Spitkovsky, and E. Waxman, Astrophys. J. Lett. 693, L127 (2009).

[28] J.-L. Vay, C. G. R. Geddes, E. Esarey, C. B. Schroeder, W. P. Leemans, E. Cormier-Michel, and D. P. Grote, Phys. Plasmas 18, 123103 (2011). 